Article original

\title{
Etude comparative entre contexte d'urgence et contexte de chirurgie programmée de l'intervention initiale des péritonites postopératoires
}

\author{
Comparative study between emergency context and programmed surgery \\ context of the initial intervention of postoperative peritonitis
}

Merouane BOUKRISSA ${ }^{1}$, Khadìdja BRAHMI-SEDDIKI ${ }^{2}$, Mohamed Amine BENHAMED ${ }^{3}$

${ }^{1}$ Service des Urgences Chirurgicales, CHU Oran

${ }^{2}$ Clinique chirurgicale A, CHU Oran

${ }^{3}$ Service de Réanimation et d'anesthésie A, CHU Oran

Auteur correspondant: mboukrissa@hotmail.com

Article reçu le 18/12/2018/Accepté le 31/12/2018

\section{MOTS CLÉS \\ Péritonite postopéra- toire, contexte d'ur- gence, reprise chirurgi- cale, CHU Oran.}

\section{Résumé}

Introduction - L'objectif de cette étude est de mettre en relief les différents profils épidémiologiques, clinico-pronostiques et thérapeutiques des péritonites postopératoires en fonction du contexte initial, d'urgence ou de chirurgie programmée, de l'intervention initiale.

Matériels et méthodes - Il s'agit d'une étude rétrospective et prospective qui a colligé 127 patients opérés pour péritonite postopératoire au service des urgences chirurgicales d'Oran. L'intervention première en contexte d'urgence avait concerné 83 patients $(65,4 \%)$ et 44 patients $(34,6 \%)$ opérés initialement dans un cadre de chirurgie programmée.

Résultats - L'âge moyen des patients opérés du groupe contexte d'urgence [GCU] était de 46,6 ans versus 49,9 ans pour les patients opérés en contexte de chirurgie programmée [GCP]. Une prédominance masculine de 59,0\% était observée dans le [GCU] versus $52,2 \%$ dans le [GCP]. Le degré de septicité des interventions, selon la classification d'Altemeier, avait montré que les interventions initiales (classes III/contaminée et IV/sale) représentaient respectivement $90,2 \%$ et $100 \%$ pour le [GCU], et $9,7 \%$ et $0 \%$ pour le [GCP]. L'état de choc était retrouvé chez 26 patients dont 17 avaient été opérés initialement en contexte d'urgence (65\%). La mortalité était de $31,3 \%$ dans le [GCU] contre $27,2 \%$ dans le [GCP]. $P=0,635$.

Conclusion - Cette étude comparative a permis de mettre en évidence les différentes caractéristiques épidémiologiques, diagnostiques et thérapeutiques distinguant les deux groupes de patients opérés selon le caractère d'urgence ou non de l'intervention première. 


\section{KEY WORDS}

Postoperative peri-
tonitis, emergency
context, surgical revi-
sion, CHU Oran.

\begin{abstract}
Introduction - The objective of this study is to highlight the different epidemiological, clinico-prognostic and therapeutic profiles of postoperative peritonitis depending on the initial context, emergency or programmed surgery, initial intervention.

Materials and methods - This is a retrospective and prospective study that gathered 127 patients operated for postoperative peritonitis in the ORAN surgical emergency department. This study is retrospective from September 2011 to September 2015 and prospective from October 2015 to December 2017. The initial intervention in an emergency context involved 83 patients (65.4\%) compared to 44 patients (34.6\%) initially operated in a planned surgery setting.
\end{abstract}

Results - The average age of patients in the Emergency Context Group [CEU] was 46.6 years versus 49.9 years for patients undergoing surgery in the context of programmed surgery [GCP]. A male predominance of $59.0 \%$ was observed in the [GCU] versus $52.2 \%$ in the [GCP]. The degree of sepsis of the interventions, according to the classification of Altemeier had shown that the initial interventions (classes III / contaminated and IV / dirty) represented respectively $90,2 \%$ and $100 \%$ for the [GCU], and $9,7 \%$ and $0 \%$ for the [GCP]. The state of shock was found in 26 patients, 17 of whom had been operated initially in emergency context $(65 \%)$. The mortality was $31.3 \%$ in the [GCU] against $27.2 \%$ in the [GCP]. $P=0.635$.

Conclusion - This comparative study made it possible to highlight the different epidemiological, diagnostic and therapeutic characteristics distinguishing the two groups of patients operated according to the emergency nature or not of the primary intervention.

\section{Introduction}

Les péritonites postopératoires sont des péritonites secondaires caractérisées par leur survenue après une intervention chirurgicale abdominale initiale qui peut être pratiquée en contexte d'urgence ou programmée ; propre ou contaminée. Plusieurs classifications ont été mises au point et la plus utilisée est celle de Hambourg[1]. Elles englobent de nombreuses pathologies qui diffèrent selon l'étiologie, le mécanisme physiopathologique, le contexte de survenue, le pronostic et le traitement.

Devant la gravité de cette complication, le chirurgien doit toujours surveiller l'apparition de moindres signes en période postopératoire afin d'éviter l'installation de défaillances d'organes. Il faut toujours avoir à l'esprit qu'une relaparotomie " blanche» vaut mieux qu'un sepsis dépassé, opéré tardivement $[2,3,4]$. L'objectif de notre travail est de mettre en exergue les différents profils épidémiologiques, clinico-pronostiques et thérapeutiques des péritonites postopératoires.

\section{Matériel et méthodes}

Il s'agit d'une étude rétrospective et prospective qui a colligé 127 patients opérés au service des urgences médico-chirurgicales d'Oran pour péritonite postopératoire (PPO). Cette étude est rétrospective allant de septembre 2011 à septembre 2015 et prospective allant d'octobre 2015 à décembre 2017.

Elle est comparative selon le contexte initial en urgence ou non de l'intervention première. L'intervention première en contexte d'urgence avait concerné 83 patients $(65,4 \%)$ et 44 patients $(34,6 \%)$ opérés initialement dans un cadre de chirurgie programmée. 


\section{Résultats}

L'âge moyen des patients opérés en contexte d'urgence était de 46,6 ans contre 49,9 ans pour les patients opérés en contexte de chirurgie programmée. Une prédominance masculine de 59,0\% était observée dans le groupe de patients opérés en contexte d'urgence [GCU] versus $52,2 \%$ dans le groupe de patients opérés en contexte de chirurgie programmée [GCP].

L'HTA, le diabète et les cardiopathies étaient réparties respectivement à $22 \%, 12 \%$ et $3 \%$ dans le [GCU] versus $20 \%, 15 \%$ et $6 \%$ dans le [GCP].

Le délai moyen de reprise chirurgicale était de 7,51 jours pour le [GCU] versus 7,95 jours pour le [GCP]. Les délais de reprise durant les 5 premiers jours étaient plus courts pour les opérés du [GCU], (voir tableau $\mathrm{N}^{\circ} 1$ ).

Tableau $N^{\circ} 1$. Répartition des délais de reprise chirurgicale selon le contexte d'urgence ou de chirurgie programmée de l'intervention initiale

\begin{tabular}{|c|c|c|}
\hline & $\begin{array}{c}\text { Chirurgie } \\
\text { d'urgence } \\
\text { (\%) }\end{array}$ & $\begin{array}{c}\text { Chirurgie } \\
\text { programmée } \\
(\%)\end{array}$ \\
\hline 5 premiers jours & 40,9 & 31,8 \\
\hline Du $6^{\text {eme }}$ au $10^{\text {eme }}$ jour & 34,9 & 45,4 \\
\hline Du $11^{\text {eme }}$ au $15^{\text {eme }}$ jour & 16,8 & 20,4 \\
\hline
\end{tabular}

La chirurgie initiale de l'étage sus mésocolique était équivalente dans les deux groupes de patients avec un taux de $27,6 \%$ pour le [GCU] versus $25 \%$ pour le [GCP].

Le degré de septicité des interventions, selon la classification d'Altemeier[5], avait montré que les interventions initiales (classes III/contaminée et IV/sale) représentaient respectivement $90,2 \%$ et $100 \%$ pour le [GCU], et $9,7 \%$ et $0 \%$ pour le [GCP] (voir tableau $\mathrm{N}^{\circ} 2$ ).

Répartition du degré de septicité selon le contexte d'urgence ou de chirurgie programmée de l'intervention initiale

\begin{tabular}{|c|c|c|c|c|c|}
\hline & $\begin{array}{c}\text { Chirurgie } \\
\text { propre }\end{array}$ & $\begin{array}{c}\text { Propre } \\
\text { contaminée }\end{array}$ & $\begin{array}{c}\text { Contami } \\
\text { née }\end{array}$ & Sale & Total \\
\hline GCP & 9 & 22 & 37 & 15 & 83 \\
\hline GCU & 3 & 37 & 4 & 0 & 44 \\
\hline Total & 12 & 59 & 41 & 15 & 127 \\
\hline
\end{tabular}

L'instabilité hémodynamique avec état de choc était retrouvée chez 26 patients dont 17 avaient été opérés initialement en contexte d'urgence $(65 \%)$. La fièvre, supérieure à $38^{\circ} \mathrm{C}$, retrouvée chez $60,2 \%$ des patients [GCU], était présente chez trois quarts des patients [GCP]. La douleur abdominale et les vomissements étaient présents respectivement chez $66,2 \%$ et $16,8 \%$ des patients [GCU] versus $59,0 \%$ et $20 \%$ des opérés [GCP]. L'arrêt du transit était présent chez $63,8 \%$ des patients [GCU] contre $59,0 \%$ [GCP] , mais la diarrhée était notée chez seulement $1 \%$ des opérés [GCU] contre $6,8 \%$ [GCP].

L'examen physique montre qu'il n'existe pas de spécificité entre les deux groupes de patients de notre étude. Cependant, l'extériorisation des viscères à l'extérieur de la plaie abdominale sous forme d'éviscération avait été le motif de reprise chez six patients sur sept opérés initialement en contexte d'urgence (voir tableau $\mathrm{N}^{\circ} 3$ ).

Tableau $N^{\circ} 3$. Répartition des signes cliniques selon le contexte d'urgence ou de chirurgie programmée de l'intervention initiale

\begin{tabular}{|l|c|c|}
\hline Signes physiques & $\begin{array}{c}\text { Chirurgie initiale } \\
\text { d'urgence } \\
(\%)\end{array}$ & $\begin{array}{c}\text { Chirurgie initiale } \\
\text { programmée } \\
(\%)\end{array}$ \\
\hline La distension abdominale & 36,1 & 36,3 \\
\hline sensibilité abdominale & 75,9 & 72,7 \\
\hline $\begin{array}{l}\text { Ecoulement anormal à } \\
\text { travers la plaie }\end{array}$ & 27,7 & 25 \\
\hline $\begin{array}{l}\text { Ecoulement anormal à } \\
\text { travers les drains } \\
\text { abdominaux }\end{array}$ & 27,7 & 15,9 \\
\hline Eviscération & 7,2 & \\
\hline
\end{tabular}

La numération formule sanguine (NFS), pratiquée chez 51 patients [GCU] $(61,4 \%)$ et 21 patients [GCP] $(47,7 \%)$, avait objectivé une hyperleucocytose chez 44 patients du [GCU] $(49,3 \%)$ et 18 patients du [GCP] $(40,9 \%)$. Le dosage de la créatininémie était réalisé chez tous les patients avec une élévation du taux de créatinine sanguine dans $26,5 \%$ des cas du [GCU] et $22,7 \%$ des cas du [GCP].

L'échographie abdominale pratiquée chez $43,3 \%$ des opérés du [GCU] et 45,4\% des patients du [GCP], avait retrouvé un épanchement péritonéal dans $34,9 \%$ des cas du [GCU] et $40,9 \%$ des cas du [GCP]. Le scanner abdominal réalisé chez $16,8 \%$ des opérés du [GCU] et $20,4 \%$ [GCP], avait objectivé un épanchement péritonéal dans $12,0 \%$ des cas du [GCU] et $20,9 \%$ des cas du [GCP].

Au cours de l'exploration chirurgicale, la nature biliodigestive du liquide péritonéal était répartie dans des proportions équivalentes dans les deux groupes. La fréquence du caractère purulent était plus importante en cas de chirurgie première programmée (voir tableau $\mathrm{N}^{\circ} 4$ ). 
Tableau $N^{\circ} 4$. Répartition de la nature du liquide péritonéal au cours de la reprise selon le contexte d'urgence ou de chirurgie programmée de l'intervention initiale

\begin{tabular}{|l|c|c|}
\hline $\begin{array}{l}\text { Nature du liquide } \\
\text { péritonéal }\end{array}$ & $\begin{array}{c}\text { Chirurgie } \\
\text { d'urgence } \\
\text { (\%) }\end{array}$ & $\begin{array}{c}\text { Chirurgie } \\
\text { programmée } \\
\text { (\%) }\end{array}$ \\
\hline biliodigestive & $26,5 \%$ & $22,7 \%$ \\
\hline purulente & $25,3 \%$ & $34,0 \%$ \\
\hline stercorale & $10,8 \%$ & $13,6 \%$ \\
\hline Louche & $14,4 \%$ & $6,8 \%$ \\
\hline séro-hématique & $16,8 \%$ & $4,5 \%$ \\
\hline
\end{tabular}

Le siège de la lésion viscérale était identifié sept fois sur 10 dans le [GCP] $(68,2 \%)$ contre environ une fois sur deux dans le [GCU] $(55,4 \%)$. L'atteinte de l'intestin grêle, du colon et du rectum, représentaient $56,3 \%$ des localisations viscérales dans le [GCU] versus $66,7 \%$ dans le [GCP]. L'atteinte gastroduodénale était de $34,5 \%$ dans le [GCU] versus $20 \%$ dans le [GCP].

Le lâchage anastomotique (ou la désunion de suture) était moins fréquent quand la chirurgie initiale était pratiquée en contexte d'urgence. Elle était observée chez 34,9\% des patients repris [GCU] versus 54,5\% [GCP]. L'atteinte du colo-rectum était deux fois plus élevée dans le [GCP]. Voir tableau $N^{\circ} 5$.

Tableau $N^{\circ} 5$. Siège de la fistule au cours de la reprise selon le contexte d'urgence ou de chirurgie programmée de l'intervention initiale

\begin{tabular}{|l|c|c|c|c|}
\cline { 2 - 5 } \multicolumn{1}{c|}{} & \multicolumn{2}{c|}{$\begin{array}{c}\text { Chirurgie initiale } \\
\text { d'urgence }\end{array}$} & \multicolumn{2}{c|}{$\begin{array}{c}\text { Chirurgie initiale } \\
\text { programmée }\end{array}$} \\
\hline Siège de la fistule & $\mathbf{N}$ & $\%$ & $\mathbf{N}$ & $\%$ \\
\hline Estomac & 5 & $6 \%$ & 1 & $2,3 \%$ \\
\hline Duodénum & 3 & $3,6 \%$ & 1 & $2,3 \%$ \\
\hline Biliaire & 8 & $9,6 \%$ & 4 & $9,1 \%$ \\
\hline Grêle & 15 & $18 \%$ & 7 & $15,9 \%$ \\
\hline Colon & 10 & $12 \%$ & 11 & $25 \%$ \\
\hline Rectum & 1 & $1,25 \%$ & 2 & $4,6 \%$ \\
\hline Utérus & 1 & $1,25 \%$ & 3 & $6,9 \%$ \\
\hline Vessie & 3 & $3,6 \%$ & 1 & $2,3 \%$ \\
\hline Autres & 37 & $44,5 \%$ & 14 & $31,8 \%$ \\
\hline
\end{tabular}

Les abcès localisés intrapéritonéaux, les textilomes, les pancréatites aigues, représentaient les autres causes d'infection abdominale postopératoire. L'abcès localisé intrapéritonéal était retrouvé deux fois plus chez les opérés du [GCU] (22\%) que chez les opérés du [GCP] (11\%).
Dans notre étude, sur les 127 cas de reprises, la cause de l'infection abdominale n'était pas retrouvée (21,2\%). Dans le [GCU] l'exploration chirurgicale n'avait pas identifié de cause d'infection abdominale postopératoire dans $23 \%$ de cas de reprises contre $18 \%$ dans le [GCP].

Le geste chirurgical réalisé, était une déconnexion de l'anastomose avec mise à la peau sous forme de stomie chez $27,7 \%$ des patients réopérés du [GCU] versus 38,6\% [GCP]. La mise en place d'une sonde de Petzer avait concerné $6 \%$ des patients du [GCU] versus 11,3\% [GCP]. La suture de la désunion avait intéressé trois patients $(3,6 \%)$ du [GCU] versus $0 \%$ du [GCP]. L'exclusion avec dérivation digestive avait été pratiquée chez $3,6 \%$ des patients du [GCU] versus 2,2\% [GCP].

La fréquence du nombre de décès dans notre étude lorsque l'intervention initiale se pratiquait en urgence était de $31,3 \%$, contre un taux de $27,2 \%$ quand la chirurgie initiale était programmée. La mortalité n'est pas significativement liée au caractère d'urgence de l'acte opératoire initial. $P=$ 0,635 .

\section{Discussion}

La moyenne d'âge de survenue des péritonites postopératoires varie, selon les séries internationales, de 34 ans à 63 ans. Dans notre série, l'âge moyen des patients des deux groupes repris pour cette infection abdominale était de 48,13 ans avec des extrêmes de 16 à 88 ans. Il était de 46,6 ans chez les patients du [GCU], légèrement plus bas que celui des patients du [GCP] 49,9 ans.

Le sexe masculin est un facteur de risque de survenue d'une péritonite postopératoire en analyse univariée $(p: 0,012)$ ainsi qu'en analyse multivariée $(O R: 2,51)$,dans une étude randomisée concernant 383 patients opérés entre 2008 et 2009 [6]. Le sexe masculin est également identifié comme facteur de risque de lâchage d'anastomose selon l'étude de Law et al [7]. En effet, sur les 196 patients qui avaient bénéficié d'une résection antérieure du rectum, la reprise chirurgicale a concerné 119 hommes et 77 femmes durant la période d'étude qui s'est étalée de septembre 1993 à novembre1998. Dans une autre étude menée par Rullier et al. [8], le risque de lâchage anastomotique était 2,7 fois plus élevé chez l'homme que chez la femme. Notre étude souligne une prédominance masculine dans le groupe [GCU] et une parité des sexes dans le [GCP].

Dans la littérature, Le contexte d'urgence de l'intervention chirurgicale initiale représente un facteur de risque de survenue de péritonite postopératoire(PPO). Les patients opérés de l'abdomen en urgence étaient plus à risque de 
développer une PPO que les patients ayant bénéficié d'une chirurgie programmée selon $\mathrm{H}$. Krukowski et al [9]. Mais ces données ne rejoignent pas les résultats de l'étude de Yoann Launay (de septembre 2006 à aout 2011) où sur 201 cas de reprises pour PPO, 34\% avaient fait l'objet d'une chirurgie initiale dans un contexte d'urgence [10].

Dans notre étude, sur les 127 patients repris pour PPO, 83 avaient été opérés initialement en contexte d'urgence, soit $65,3 \%$. Ainsi, nous rapportons une fréquence de survenue de PPO multipliée par 2 en cas de chirurgie initiale en contexte d'urgence. Ce risque est multiplié par quatre dans l'étude malienne de F.L.Simoto Notue[11].

\section{Le caractère septique}

Les facteurs favorisants la survenue d'une infection abdominale postopératoire sont les interventions effectuées dans un contexte septique, en situation d'urgence ou chez un patient à risque d'immunodépression. Les interventions effectuées dans un contexte initial septique prédisposent à la survenue d'un sepsis postopératoire. Il a été rapporté une augmentation de l'incidence des PPO de $0,1 \%$ après chirurgie propre à $6,5 \%$ en cas d'une chirurgie septique $[12,13]$. De même que dans une étude danoise, Bremmelgaard et al ont rapporté une incidence croissante des complications infectieuses postopératoires avec une variabilité interopérateur de 1,4 à 13,2 \% en chirurgie générale contaminée et de 15,4 à $23,5 \%$ en chirurgie septique [14].

Les conditions opératoires et le déroulement de la chirurgie ont également un impact sur le risque de fistule. Dans l'étude d'Alvès \& coll, il a été rapporté que la chirurgie digestive en condition septique, les difficultés rencontrées lors de la réalisation de l'anastomose, les anastomoses colocoliques augmentent le risque de fistule anastomotique $[15,16]$.

Hormis les anastomoses colocoliques, les anastomoses basses sont aussi plus à risque de fuir [17-19]. Dans une série de 196 patient réopérés pou PPO, Y. Marzougui et al.[20], 49,01\% de cas d'interventions initiales étaient de classes 3 et 4 . Dans l'étude de F.l Simo Notue, 68,8\% étaient des actes initiaux de classes 3 et 4 [11].

Dans notre étude, le caractère septique de l'intervention initiale est significativement lié au contexte d'urgence. La totalité des chirurgies «sales» de classe 4 d'Altemeier et la quasi-totalité des patients de classe 3 (neuf patients sur 10), avaient été opérés initialement en urgence. Il en résulte que $93 \%$ des chirurgies de ces deux classes 3 et 4 avaient été réalisées dans un conteste d'urgence.

\section{Les délais de reprise chirurgicale}

Les péritonites postopératoires sont tardivement reconnues, classiquement entre le cinquième et le septième jour postopératoire. Un second pic correspond aux complications retardées constatées au-delà de la deuxième semaine. Le délai moyen de réintervention était de 9,53 jours dans l'étude de l'AFC et de 9 jours dans l'étude de T.Bensignor [21]. Ce délai était compris entre le 5ème et le 7ème jour dans une étude américaine de Neil H.Hyman en 2007[22]. Dans notre étude, le délai moyen de reprise chirurgicale de notre cohorte de malades était de 7,67 jours.

Le contexte d'urgence de l'intervention initiale ne semble pas influencer le délai de reprise qui était de 7,51 jours. Néanmoins, des délais plus courts, durant les 5 premiers jours, sont notés chez $40,9 \%$ des patients du [GCU] versus $31,8 \%$ du [GCP], en raison de l'apparition précoce de critères cliniques de reprise chirurgicale, notamment l'écoulement anormal à travers les drains dans le [GCU].

\section{Les manifestations cliniques}

La défaillance hémodynamique conditionne le pronostic. Dans l'étude française de T. Bensignor concernant 191 reprises pour PPO, 16,5\% des patients avaient présenté un état de choc. Dans notre cohorte, environ un patient sur cinq avait présenté un état de choc $(20,5 \%)$ dont les trois quarts avaient nécessité l'administration de catécholamines. Mais l'instabilité hémodynamique avec état de choc était retrouvée chez deux patients sur trois qui étaient opérés initialement en contexte d'urgence. II s'agissait de patients opérés initialement de péritonites aigues généralisées, d'infarctus mésentériques qui présentaient des signes de choc septique.

La fièvre est l'un des signes les plus fréquents [23]. Dans une étude prospective de Le Gall et al [24], une fièvre supérieure à $39{ }^{\circ} \mathrm{C}$ entre le troisième et le dixième jour postopératoire traduit dans $66 \%$ des cas une affection intrapéritonéale. De même que l'intensité de la fièvre $\left(\geq 38^{\circ} 5 \mathrm{C}\right)$ pourrait être un élément d'orientation [25]. Dans notre travail, la fièvre supérieure à $38^{\circ} \mathrm{C}$, était présente chez $60,2 \%$ des patients [GCU] versus $75 \%$ des patients [GCP] et ne semble pas être un critère significativement spécifique du [GCP].

En période postopératoire, la douleur abdominale, l'iléus et les vomissements sont des signes cliniques difficiles à interpréter chez l'opéré récent. La douleur et le ballonnement sont respectivement présents dans $44 \%$ et $42 \%$ des cas dans l'étude de Levy [23]. A travers notre travail, la douleur abdominale, l'arrêt du transit et les vomissements ne sont pas significativement liés au contexte d'urgence de l'intervention première. Cependant la diarrhée, présente chez $6,8 \%$ [GCP] contre seulement $1 \%$ des opérés [GCU] traduit 
le lâchage d'une anastomose colique ou rectale pratiquée en contexte de chirurgie programmée. De même qu'à l'examen physique, il n'y avait pas de spécificité entre les deux groupes de patients de notre étude. Cependant, l'extériorisation des viscères à l'extérieur de la plaie abdominale sous forme d'éviscération avait été le motif de reprise chez six patients sur sept opérés initialement en contexte d'urgence.

\section{Les données biologiques et morphologiques}

En période postopératoire, les examens biologiques sont contributifs dans le diagnostic de défaillance d'organes, cependant, ils le sont moins dans le diagnostic de péritonite postopératoire. A travers notre travail, l'hyperleucocytose et l'augmentation de la créatininémie n'étaient pas significativement liées au contexte d'urgence de l'intervention initiale.

Le scanner abdominal, examen de référence dans l'exploration de la cavité abdominale en période postopératoire, avait été réalisé chez $64,9 \%$ des patients, dans la série de T. Bensignor, et avait objectivé dans $59,7 \%$ des cas un épanchement péritonéal. Dans notre étude, bien que l'exploration échographique ait été réalisée trois fois plus que le scanner abdominal, il apparait que la pratique de ces examens ainsi que la découverte d'épanchement intrapéritonéal ne soit pas spécifique à l'un des deux groupes étudiés.

\section{Les données de prise en charge chirurgicale}

L'étiologie la plus fréquente retrouvée dans la majorité des études est le lâchage d'une anastomose ou la désunion d'une suture dans 40 à $72 \%$ des cas selon les séries [12, 2628]. Ce taux de lâchage était de $51,2 \%$ dans une étude multicentrique réalisée par l'AFC [29] (2010), de 49\% dans l'étude de A. Hajar (2017) [30]et de 40\% dans la série française de Y. Launey (2017) [10]. Notre étude, à travers les 127 patients repris pour PPO, avait retrouvé un taux de lâchage anastomotique chez quatre patients sur dix. Le lâchage anastomotique (ou la désunion de suture) était moins fréquent quand la chirurgie initiale était pratiquée en contexte d'urgence. L'atteinte du colo-rectum était deux fois plus élevée dans le [GCP]. Il s'agissait notamment de résections chirurgicales de cancers du colon et du rectum. Bien que la répartition des deux groupes de notre étude soit équivalente à travers les étages sus-mésocolique et sous-mésocolique, L'atteinte gastroduodénale était une fois et demie plus importante dans le [GCU]. L'atteinte de l'intestin grêle, du colon et du rectum était équivalente dans les deux groupes.

L'abcès localisé intrapéritonéal était retrouvé deux fois plus chez les opérés du [GCU] que chez les opérés du [GCP]. Ces résultats seraient expliqués par le caractère septique des interventions premières pratiquées dans un contexte d'urgence.
L'exploration chirurgicale n'avait pas identifié de cause d'infection abdominale postopératoire dans $23 \%$ de cas de reprises du [GCU] contre $18 \%$ dans le [GCP]. Le contexte de l'intervention initiale ne semble pas influencer la non identification de la cause de PPO.

Dans la majorité des cas, dans un site péritonéal infecté, à chaque fois que se présentait un lâchage d'anastomose ou une désunion de suture, l'abouchement à la peau était pratiqué quand la mobilité du segment digestif le permettait. Dans l'étude de Y. Launey et al, la prise en charge peropératoire des lésions a été définie comme la réalisation d'une iléostomie ou d'une colostomie en cas de lésion infra-mésocolique ou un drainage en cas de lésion supra-mésocolique[10].

La déconnexion de l'anastomose avec mise à la peau sous forme de stomie avait été réalisée moins frequement chez des patients du [GCU] en raison du site de la source infectieuse, gastroduodénal qui était plus fréquent. Pour rappel, le lâchage anastomotique des segments digestifs de l'étage sus mésocolique rend souvent l'abouchement à la peau impossible. A défaut, plusieurs techniques ont été pratiquées en fonction du stade avancé de l'infection abdominale, de l'importance et du siège des lésions. Il s'agissait de réaliser le plus souvent l'intubation de la brèche par une sonde de Petzer avec drainage en regard systématique, ou de suturer la brèche et y associer un double-drainage en regard ou plus rarement réaliser une exclusion digestive avec fermeture du bout distal (duodénal).

\section{Conclusion}

Le contexte d'urgence de l'intervention chirurgicale initiale représente un facteur de risque de survenue de péritonite postopératoire(PPO)[9]. A travers cette étude, il existe une prédominance masculine dans le [GCU]. De même que le caractère septique de l'intervention initiale est significativement lié au contexte d'urgence. En postopératoire, l'installation d'un état de choc ou la découverte d'une éviscération sont observées notamment chez les patients opérés initialement en urgence. La diarrhée est un signe présent principalement chez les patients du [GCP]. En peropératoire, le lâchage anastomotique est plus fréquent dans le $[\mathrm{GCP}]$ et la découverte d'abcès intrapéritonéal isolé est plus fréquente dans le [GCU].

\section{Conflits d'intérêt}

Les auteurs déclarent n'avoir aucun conflit d'intérêt. 


\section{Références bibliographiques}

[1]. Wittmann, D.H., Intraabdominal infections-introduction. World journal of surgery, 1990. 14(2): p. 145-147.

[2]. Mariette, C., Principes de prise en charge chirurgicale des péritonites postopératoires. Journal de Chirurgie, 2006. 143(2): p. 84-87.

[3]. Montravers, P., et al., Guidelines for management of intra-abdominal infections. Anaesthesia Critical Care \& Pain Medicine, 2015. 34(2): p. $117-130$

[4]. Sartelli, M., et al., 2013 WSES guidelines for management of intra-abdominal infections. World Journal of Emergency Surgery, 2013. 8(1): p. 3.

[5]. Altemeier WA, Burke JF, Puitt BA, Sandusky WR. Manual on control of infection in surgical patients. JB Lippincott 2ème Ed, Philadelphia, 1984, p 29

[6]. Jeong, O., et al., Effect of age on surgical outcomes of extended gastrectomy with D2 lymph node dissection in gastric carcinoma: prospective cohort study. Annals of surgical oncology, 2010. 17(6): p. 15891596.

[7]. Szeto, C.-C., et al., Recurrent and relapsing peritonitis: causative organisms and response to treatment. American Journal of Kidney Diseases, 2009. 54(4): p. 702-710.

[8]. Montravers, P., et al., Dynamic changes of microbial flora and therapeutic consequences in persistent peritonitis. Critical Care, 2015. 19(1): p. 70.

[9]. Krukowski, Z. and N. Matheson, Ten-year computerized audit of infection after abdominal surgery. British Journal of Surgery, 1988. 75(9): p. 857-861.

[10]. Launey, Y., et al., Risk factors for mortality in postoperative peritonitis in critically ill patients. World journal of critical care medicine, 2017. 6(1): p. 48.

[11]. Laurence, M.S.N.F., peritonites postoperatoires: diagnostic et traitement en chirurgie generale au chu gabriel toure. 2014.

[12]. Roehrborn, A., et al., The microbiology of postoperative peritonitis. Clinical Infectious Diseases, 2001. 33(9): p. 1513-1519.

[13]. Montravers, P., L. El Housseini, and R. Rekkik, Les péritonites postopératoires: diagnostic et indication des réinterventions. Réanimation, 2004. 13(6-7): p. 431-435.

[14]. Bremmelgaard, A., et al., Computer-aided surveillance of surgical infections and identification of risk factors. Journal of Hospital Infection, 1989. 13(1): p. 1-18.

[15]. Szeto, C.-C., et al., Recurrent and relapsing peritonitis: causative organisms and response to treatment. American Journal of Kidney Diseases, 2009. 54(4): p. 702-710.

[16]. Brandstrup, B., et al., Effects of intravenous fluid restriction on postoperative complications: comparison of two perioperative fluid regimens: a randomized assessor-blinded multicenter trial. Annals of surgery, 2003. 238(5): p. 641.

[17]. Akiyoshi, T., et al., Incidence of and risk factors for anastomotic leakage after laparoscopic anterior resection with intracorporeal rectal transection and double-stapling technique anastomosis for rectal cancer. The American journal of surgery, 2011. 202(3): p. 259-264.

[18]. Rullier, E., et al., Risk factors for anastomotic leakage after resec- tion of rectal cancer. BJS, 1998. 85(3): p. 355-358.

[19]. Matthiessen, P., et al., Risk factors for anastomotic leakage after anterior resection of the rectum. Colorectal Disease, 2004. 6(6): p. 462-469.

[20]. Marzougui, Y., et al., Postoperative peritonitis: pronostic factors of mortality. Archives de l'Institut Pasteur de Tunis, 2014. 91(1-4): p. 67-76.

[21]. Bensignor, T., Prise en charge chirurgicale des péritonites postopératoires apres chirurgie digestive: étude rétrospective sur 191 patients. [22]. Neil Hyman, et al. Anastomotic Leaks After Intestinal Anastomosis : It's Later Than You ThinkAnn Surg. 2007 Feb; 245(2): 254-258. doi: [10.1097/01.sla.0000225083.27182.85].

[23]. Levy, E., et al. Péritonites post-opératoires: données communes. in Annales de chirurgie. 1985. Elsevier.

[24]. Le Gall, J., et al., Diagnostic features of early high post-laparotomy fever: A prospective study of 100 patients. British Journal of Surgery, 1982. 69(8): p. 452-455.

[25]. Kron, I.L., P.K. Harman, and S.P. Nolan, The measurement of intra-abdominal pressure as a criterion for abdominal re-exploration. Annals of surgery, 1984. 199(1): p. 28.

[26]. Montravers, P., et al., Emergence of antibiotic-resistant bacteria in cases of peritonitis after intraabdominal surgery affects the efficacy of empirical antimicrobial therapy. Clinical Infectious Diseases, 1996. 23(3): p. 486-494.[27]. Guivarc'h, M., D. Houssin, and A. Chapman. 100 cases of generalised postoperative peritonitis (author's transl). in Annales de chirurgie. 1977.

[28]. Dupont, H., et al., Can yeast isolation in peritoneal fluid be predicted in intensive care unit patients with peritonitis? Critical care medicine, 2003. 31(3): p. 752-757.

[29]. Péritonites postopératoires - B. Heyd, J.-G. Balique, N. Dehni, éditeur Arnette, collection AFC, livre neuf année 2010, isbn 9782718412399.

[30]. ADIL, H., Facteurs prédictifs de mortalité dans les péritonites postopératoires: Etude rétrospective à propos de 100 cas, 2017. 
